\title{
Carbapenem-Resistant Enterobacterales: Considerations for Treatment in the Era of New Antimicrobials and Evolving Enzymology
}

\author{
Maxwell J. Lasko ${ }^{1}$. David P. Nicolau ${ }^{1,2}$ \\ Published online: 7 February 2020 \\ (C) Springer Science+Business Media, LLC, part of Springer Nature 2020
}

\begin{abstract}
Purpose of Review Gram-negative resistance is a growing concern globally. Enterobacterales, formerly Enterobacteriaceae, have developed resistance mechanisms to carbapenems that leave very few antimicrobial options in the clinician's armamentarium.

Recent Findings New antimicrobials like ceftazidime-avibactam, meropenem-vaborbactam, imipenem-relebactam, cefiderocol, and plazomicin have the potential to overcome resistance mechanisms in Enterobacterales including different classes of carbapenemases.

Summary Novel $\beta$-lactam/ $\beta$-lactamase inhibitors, plazomicin, and cefiderocol give the clinician options that were once not available. Utilizing these options is of the utmost importance when treating carbapenem-resistant Enterobacterales.
\end{abstract}

Keywords Resistance $\cdot$ Carbapenemase $\cdot$ Enterobacterales $\cdot$ Enterobacteriaceae

\section{Introduction}

Antimicrobial resistance is one of the 2019 World Health Organization's (WHO) top ten threats to global health [1]. With increasing exposures to antimicrobials, organisms that were once easily treatable now harbor mechanisms of resistance that leave few treatment options for clinicians. Carbapenem-resistant Enterobacterales, formerly known as Enterobacteriaceae, are a group of organisms that are among the most difficult to treat; therefore, it is no surprise that the CDC considers them to be one of the top three most urgent

This article is part of the Topical Collection on Antimicrobial Development and Drug Resistance

David P. Nicolau

david.nicolau@hhchealth.org

Maxwell J. Lasko

Maxwell.Lasko@hhchealth.org

1 Center for Anti-Infective Research and Development, Hartford Hospital, 80 Seymour Street, Hartford, CT 06102, USA

2 Division of Infectious Diseases, Hartford Hospital, Hartford, CT, USA threats with resistance spreading rapidly in the USA and throughout the world [2]. As of 2018, the CDC has reported at least one type of carbapenemase in all 50 states, and rarer genotypic expressions are becoming more common [3]. Prior to the introduction of novel $\beta$-lactam $/ \beta$-lactamase inhibitors with activity against carbapenemase producers, mortality rates varied from $24 \%$ to as high as $74 \%[4,5]$. It is imperative that clinicians understand definitions of carbapenem-resistant Enterobacterales (CRE), local resistance patterns, and how to optimize treatment based on infection site.

There are major oversights when discussing carbapenemresistant organisms (CRO) or CREs. For one, CRE's simplified definition of Enterobacterales resistant to carbapenems does an injustice to the complexities of developed mechanisms of resistance [6]. Combinations of extended spectrum $\beta$-lactamase (ESBL), overexpression of ampC and loss of porin channels may confer resistance to ertapenem but meropenem or imipenem may remain active [7-9]. While considered to be CRE using conventional definitions, these non-carbapenemase-producing organisms may be amenable to high-dose carbapenem therapy and do not pose the same potential for transmissibility due to their lack of plasmid conferred resistance [10]. In contrast to these non-carbapenemaseproducing organisms, it is important to delineate if CRE are 
carbapenemase producers as differing enzymes will have variable phenotypic profiles to commercially available antimicrobials as well as a high degree of transmissibility due to their plasmid-mediated resistance profiles. OXA-48, a serine-based carbapenemase, may hydrolyze carbapenems, yet ceftazidime and cefepime will retain activity if no other mechanisms of resistance are expressed $[11,12]$. Isolates may and often have additional mechanisms of resistance included with carbapenemase. Additional subtleties with carbapenem resistance occur demonstrating the need for more specific terminology. Moreover, the likelihood of different types of resistance varies between geographical regions. North America and Israel tend to have a higher prevalence of Klebsiella pneumoniae carbapenemase (KPC) whereas metallo- $\beta$-lactamase (MBL) can be found in India, Italy, and Greece [7, 13-17]. OXA carbapenemases are endemic to Europe, Northern Africa, and the Middle East [7, 11, 12, 14]. A potential treatment option for a KPC may not necessarily treat an MBL and can be overlooked using broad terminology like CRE. Table 1 highlights different groups and classifications of $\beta$-lactamase including potential carbapenemase producers.

Perhaps the greatest disservice when discussing treatment of CRE is assuming the clinician knows the organism prior to starting empiric therapy. Many risk factors associated with the development of CRE are associated with other multidrugresistant nosocomial infections like Pseudomonas aeruginosa [18]. Empiric drug choice in an at-risk septic patient should take into account the roles and limitations of each therapy option. The remainder of this review will compare antimicrobials to treat CRE and discuss trends in treatment management of this infection entity.

\section{Polymyxins}

After the introduction of polymyxin B and colistin (polymyxin E) in the 1950s, they quickly fell out of favor due to their high risk of acute kidney injury (AKI) and unpredictable kinetics [19]. With the emergence of multidrug-resistant organisms like CRE, the polymyxin class has re-emerged as a backbone of salvage therapy prior to the clinical availability of novel $\beta$-lactam $/ \beta$-lactamase inhibitors (BL/BLI) targeted against carbapenemases. An international guideline on polymyxin published in 2019 attempts to promote the optimal use of these drugs [20]. While this document presents a contemporary overview of the polymyxins and intends to promote strategies to improve outcome while minimizing the toxicodynamic profile of the class, it is important to note that a majority of guidance is based on small theoretical models, and for several pieces, such as polymyxin use in CRE, the committee did not reach a unanimous consensus.

Polymyxin B and colistin are fairly similar chemically even though their pharmacokinetic parameters differ substantially. Both agents have a similar mechanism of action by puncturing and disrupting the outer membrane integrity leading to bactericidal activity and possible synergy with other antimicrobials [19]. These agents also demonstrate AUC/MIC pharmacodynamics with a targeted average steady state $\left(\mathrm{C}_{\mathrm{ss} \text { avg }}\right)$ of $2 \mathrm{mg} / \mathrm{L}$ [20].

Colistin is administered as pro-drug colistin methanesulfonate (CMS) or colistimethate, and the conversion from inactive to active drug is highly variable. It may take up to several hours to achieve adequate concentrations of drug even with appropriate loading doses. Unfortunately, inadequate rapid concentrations of colistin in the critically ill have demonstrated increased risk of 30-day mortality and may breed polymyxin resistance which is incredibly concerning considering patient frailty [19-24]. Both the active and non-active forms of colistin are highly excreted into the urine, and the inactivated CMS is partially converted to colistin allowing the drug to reach higher concentrations in the urine than Polymyxin B [19]. Colistin dosing units can be expressed as milligrams of colistin-based activity (CBA) or international units (IU) depending on country. Approximately one million $\mathrm{IU}$ is equivalent to $33 \mathrm{mg}$ of CBA.

Polymyxin B is formulated in its active sulfate form, and it is not actively secreted in the urine. The preferable pharmacokinetics and decreased risk of AKI often make polymyxin B the preferred polymyxin for non-urinary systemic infections $[19,20,25]$. A loading dose of $2.0-2.5 \mathrm{mg} / \mathrm{kg}$ of total body weight followed by a maintenance dose of $1.25-1.5 \mathrm{mg} / \mathrm{kg}$ of total body weight divided into 2 dose $12 \mathrm{~h}$ apart is recommended without renal dosage adjustment $[25,26]$. Neither parental polymyxin B nor colistin achieve adequate lung concentration and are suboptimal choices for the treatment of respiratory infection as monotherapy [19].
Table 1 Classification of $\beta$ lactamase

\begin{tabular}{|c|c|c|c|}
\hline $\begin{array}{l}\text { Ambler molecular } \\
\text { classification }\end{array}$ & $\begin{array}{l}\text { Busch-Jacoby } \\
\text { functional groups }\end{array}$ & $\begin{array}{l}\text { Active binding } \\
\text { site }\end{array}$ & Common enzymes \\
\hline A & $2 \mathrm{a}, 2 \mathrm{~b}, 2 \mathrm{be}, 2 \mathrm{br}, 2 \mathrm{c}, 2 \mathrm{e}, 2 \mathrm{f}$ & Serine & TEM, SHV, KPC, CTX-M, GES \\
\hline B & 3 & Metallo & $V I M, N D M, I M P$ \\
\hline $\mathrm{C}$ & 1 & Serine & AmpC, P99 \\
\hline $\mathrm{D}$ & $2 \mathrm{~d}, 2 \mathrm{de}, 2 \mathrm{df}$ & Serine & $O X A$ \\
\hline
\end{tabular}

Italicized enzymes represent carbapenemase production 
The polymyxins should generally be given in combination with another susceptible or synergistic antimicrobial when treating CRE due to the high potential for failure, variable pharmacokinetics, and development of resistance observed on monotherapy. As novel therapies active against carbapenemase-producing organisms become more widely available, the use of polymyxins as the backbone of treatment for CRE should subside and the role of these agents should be reserved as salvage therapy when all other options have been exhausted.

\section{Aminoglycosides}

Similar to polymyxins, aminoglycosides were first clinically used in the 1940s and were a mainstay of therapy until the 1980s with the invention of safer antimicrobials like cephalosporins, carbapenems, and fluoroquinolones. Aminoglycosides carry an increased risk of nephrotoxicity and ototoxicity compared with the antimicrobials mentioned above [27]. Higher rates of adverse events occur with more frequent dosing, and a modified once-daily dosing regimen as demonstrated in the Hartford nomogram may mitigate these risks [28]. Aminoglycosides can play a crucial role in CRE because their mechanism of action is not affected by carbapenemases; however, many CRE organisms harbor aminoglycoside modification enzymes (AME) $[29,30]$. As such, the therapeutic role of conventional aminoglycosides, like gentamicin and amikacin, can be highly variable based on the widespread geographic distribution of AMEs.

Plazomicin (Zemdri®) is a new aminoglycoside that is synthetically derived from sisomicin which remains stable against most AMEs and it has been shown to be synergistic when given in combination with $\beta$-lactams in vivo [31-33]. A phase III trial (CARE study) attempted to demonstrate efficacy of plazomicin combination therapy compared with polymyxin combination therapy for a multitude of carbapenemresistant Enterobacterales infections [34]. Although the study did not reach an adequate power due to slow enrollment into the study, researchers reported a descriptive decrease in allcause mortality. Fifteen patients who received plazomicin combination therapy were further evaluated to determine if adequate concentrations of each drug were achieved [35]. Of the 15 subjects treated with plazomicin combination, $0 \%$ treated with plazomicin and meropenem reached a meropenem $f \mathrm{~T}$ $>$ MIC of $40 \%$. Furthermore, only $75 \%$ of subjects treated with combination plazomicin and tigecycline reached tigecycline $f \mathrm{AUC} / \mathrm{MIC}$ concentrations of $0.9 \mathrm{mg} / \mathrm{L}$ or greater, yet $83 \%$ of subjects with inadequate meropenem or tigecycline concentrations achieved microbiological response. These data demonstrate the potential clinical utility of plazomicin in the treatment of CRE.
While plazomicin utilizes the once-daily dosing regimen to improve efficacy and minimize toxicity, dose adjustments are required in patients with decreased renal function. Asempa and colleagues evaluated a group of 37 enrolled patients being treated for CRE bloodstream infections or pneumonia, who were originally enrolled in the CARE study [36]. Plazomicin blood samples were collected $10 \mathrm{~h}$ post dose to determine an appropriate dosing interval based on the Hartford nomogram. Afterwards, the information was simulated to predict toxic trough concentrations greater than $3 \mathrm{mg} / \mathrm{L}$ and an efficacious interval-normalized AUC. Use of the nomogram reduced the incidence of potentially toxic trough concentrations while maintaining similar target AUCs. Additionally, the application of the Hartford nomogram appears useful in less sick patients with UTIs [37].

While limited clinical data exist thus far, the microbiologic potency, pharmacokinetic, and pharmacodynamic profiles of plazomicin suggest that this agent is a viable option for CRE in combination with conventional compounds or the novel BL/BLIs. Other examples of plazomicin's utility include in combination to treat an MBL producer where novel BL/ BLIs lack in vitro activity, patients with a hypersensitivity to ceftazidime and carbapenems, or patients requiring a oncedaily injection being treated in an outpatient antimicrobial treatment program.

\section{Double Carbapenem}

As mentioned above, KPC is the most prevalent carbapenemase produced in the USA, and antimicrobial options were often scarce prior to the introduction of novel $\beta$ lactam $/ \beta$-lactamase inhibitors. Bulik and Nicolau proposed the use of combination ertapenem and high-dose doripenem or meropenem as a method to combat CRE [38]. The theoretical mechanism uses ertapenem as a suicide molecule with high affinity for KPC enzymes, allowing meropenem or doripenem to treat the organism. Both their in vitro model and murine thigh model demonstrated bacterial reduction with a roughly $3 \log$ and $1 \log$ reduction, respectively. Clinical studies and case reports have also demonstrated an increase in microbiological cure and a decrease in all-cause mortality compared with other options like polymyxins [39-42]. While the clinical utility of double carbapenem therapy has demonstrated in patients with CRE, it is important to recognize that the efficacy of this regimen is restricted to KPC-based infections.

\section{B-Lactam/B-Lactamase Inhibitors}

Novel $\beta$-lactam $/ \beta$-lactamase inhibitors are a revolutionary treatment option for carbapenemase-producing 
Enterobacterales. Ceftazidime-avibactam (Avycaz $\left.{ }^{\circledR}\right)$, meropenem-vaborbactam (Vabomere ${ }^{\circledR}$ ), and the new BL/ BLI imipenem-relebactam (Recarbrio $\left.{ }^{\circledR}\right)$ have brought welcomed treatment options to clinicians treating these MDR organisms. It is important to note that these drugs do not all cover the same carbapenemase. Table 2 highlights the differences in activity against Ambler classification of carbapenemases and the Clinical Laboratory and Standards Institute (CLSI) MIC breakpoints for novel BL/BLIs as well as novel antimicrobials that will be discussed.

Avibactam, a novel diazabicyclooctane $\beta$-lactamase inhibitor, restores ceftazidime activity against group $\mathrm{A}$, and some $\mathrm{D}$ (OXA-48) serine carbapenemases [43, 44]. There is no ceftazidime restoration against class B metallo- $\beta$-lactamases; however, there are several successful case reports of using ceftazidime-avibactam with aztreonam for the treatment of New Delhi metallo- $\beta$-lactamase (NDM), and aztreonamavibactam is currently in clinical development [45-47]. Ceftazidime-avibactam has displayed consistently improved outcomes relative to polymyxin-based regimens as numerous reports show decreased all-cause mortality, increased microbiologic cure, and reduced toxicity [48-53].

At present the vast majority of clinical and microbiologic outcome data with these new BL/BLIs for CRE-based infections accumulated during the clinical utilization of ceftazidime-avibactam, where this new agent is often being used as salvage therapy in patients failing polymyxin-based regimens. As a result, it is not surprising that treatment failure and the development of resistance on therapy have been observed in these pre-selected salvage use scenarios. Specifically, the emergence of a mutant KPC-3 variant has been noted during therapy [54]. While resistant to ceftazidime-avibactam, the organism's genotype reverted to resemble that of an ESBL testing susceptible to meropenem and not surprisingly to other BL/BLIs like meropenem-vaborbactam [55]. Shields and colleagues also proposed ceftazidime-avibactam treatment failure correlates to patients treated for healthcare-associated pneumonias and continuous renal replacement therapy [56]. Their single-center retrospective study looked at treatment failure with ceftazidime-avibactam and found that $60 \%$ of clinical failures were treated for pneumonia compared with $29 \%$ of successes ( $p=0.045$, OR 3.09, 1.03-9.34). Similar success and failure trends were seen with continuous renal replacement therapy at $7 \%$ and $37 \%$ respectively $(p=0.046$, OR $4.78,95 \%$ CI 1.03-22.2). This raises the question whether resistance and treatment failure were observed because of inappropriate dosing in these critically ill patients. This controversy with ceftazidime-avibactam highlights that it is crucial that patients receiving these novel BL/BLIs are aggressively dosed with optimal exposures to achieve clinical cure.

Vaborbactam, another non- $\beta$-lactam $/ \beta$-lactamase inhibitor, restores meropenem against Ambler group $A$ and $C \beta$ lactamase $[55,57]$. The addition of a boron structure allows for higher affinity for KPC-producing Enterobacterales, but has no effect on other MDR like Acinetobacter baumannii or P. aeruginosa. Vaborbactam, furthermore, does not restore meropenem activity against group B or D carbapenemases.

Targeting Antibiotic Non-susceptible Gram-negative Organisms (TANGO-II) trial evaluated meropenemvaborbactam against best available therapy (either monotherapy or combination of polymyxin, colistin, high-dose meropenem, ceftazidime-avibactam $(n=1)$, or aminoglycoside) for the treatment of CRE infections [58]. Eligible patients were enrolled from 8 countries where KPC is prevalent. It is important to note that a majority of isolates grew Klebsiella pneumoniae (87.2\%), and the major sites of infection were bacteremia (46.8\%), followed by complicated urinary tract infection (34\%), healthcare- and ventilatorassociated pneumonia (10.6\%), and complicated intraabdominal infection (8.6\%). All patients with previous antimicrobial failure were placed in the meropenem-vaborbactam group. Meropenem-vaborbactam had statistically significant

Table 2 Novel antimicrobial Ambler class activity

\begin{tabular}{|c|c|c|c|c|c|}
\hline Drug & Ambler group A & Ambler group B & Ambler group $\mathrm{C}$ & Ambler group D & $\begin{array}{l}2019 \text { CLSI Enterobacterales } \\
\text { MIC breakpoint }\end{array}$ \\
\hline \multicolumn{6}{|c|}{$\beta$-lactam $/ \beta$-lactamase inhibitor } \\
\hline Ceftazidime-avibactam & + & - & + & $+(\mathrm{OXA}-48)$ & $8 / 4$ \\
\hline Meropenem-vaborbactam & + & - & + & - & $4 / 8^{*}$ \\
\hline Imipenem-relebactam & + & - & + & - & $1 / 4 * *$ \\
\hline \multicolumn{6}{|c|}{ Other novel antimicrobials } \\
\hline Plazomicin & + & + & + & + & $2 * *$ \\
\hline Cefiderocol & + & + & + & + & $2 * *$ \\
\hline Fosfomycin & + & + & + & + & N/A \\
\hline
\end{tabular}

*Higher than meropenem breakpoint, avoid use in OXA endemic areas

**FDA minimum inhibitory concentration breakpoint

$O X A$, oxacillinase; CLSI, Clinical and Laboratory Standards Institute 
improvement for clinical cure at end of treatment (ARR $32.3 \%, 95 \%$ CI $3.3-61.3, p=0.03$ ) and at test of cure (ARR $32.7 \%, 95 \%$ CI $4.6-60.8, p=0.02)$. There was no difference in 28-day all-cause mortality; however, when previous treatment failure was removed, meropenem-vaborbactam did show statistical difference (absolute reduction 29\%, 95\% CI 54.3-3.7, $p=0.02$ ). Drug-related adverse events, including nephrotoxicity, were seen less often in the meropenemvaborbactam-treated patients $(24 \%)$ than in best available therapy $(44 \%)$.

Of concern with meropenem-vaborbactam is that the current CLSI-/FDA-approved breakpoint for Enterobacterales is $\leq 4 / 8$. This encompasses $30-40 \%$ of the MIC distribution for OXA-producing organisms despite the designation of no in vitro activity for this class of carbapenemases as mentioned in the package insert of the commercially available product. Despite no activity and no clinical data, the elevated breakpoint relative to that of meropenem at $\leq 1$ is due solely to the nuances in dosing between the products [59]. As a result, caution should be exercised when using meropenemvaborbactam for the treatment of susceptible carbapenemaseproducing Enterobacterales when OXA-based enzymes are in the differential and genotypic profiling has not confirmed the enzymologic driver of resistance.

Relebactam, like avibactam, is a diazabicyclooctane $\beta$ lactamase inhibitor restoring imipenem against group $\mathrm{A}$ and $\mathrm{C} \beta$-lactamases $[60,61]$. In vitro and animal models show efficacy with imipenem-relebactam, and it has recently been approved for complicated urinary tract infections and complicated intra-abdominal infections [60-66]. Motsch and colleagues recently published a randomized controlled trial comparing imipenem-relebactam to imipenem and colistin for imipenem non-susceptible bacterial infections [67]. Out of the 31 modified microbiologic intent to treat population, a majority of isolates were $P$. aeruginosa, (77\%) followed by Klebsiella spp. (16\%) and other Enterobacterales (6\%). Imipenem-relebactam was considered non-inferior for microbiological cure $(71 \%)$ compared with imipenem and colistin (70\%) and had a significant 28-day favorable response (71\% vs. $40 \%, 90 \%$ CI $1.3-51.5)$. The authors further described a non-significant reduced 28-day all-cause mortality of $10 \%$ with imipenem-relebactam compared with $30 \%$ of imipenem and colistin subjects. Notably, CRE specific data demonstrated only one out of four carbapenem-resistant Enterobacterales showing a microbiological cure compared with one out of one imipenem and colistin treated subject. Authors attributed microbiologic failure of imipenem-relebactam to organisms harboring several group $\mathrm{A}$ and $\mathrm{C}$ extended $\beta$-lactamases. The therapeutic niche of imipenem-relebactam remains unclear as it may be used as an additional option for CRE or it may be more useful in treating multidrug-resistant $P$. aeruginosa including those producing carbapenemases [62]. Similarly to ceftazidime-avibactam, the additional pseudomonal coverage may make imipenem-relebactam an appropriate option for empiric therapy in a septic patient with high risk of CRE and pseudomonal infection. Unlike the two previously approved $\beta$-lactam/ $\beta$-lactamase inhibitors which are recommended to have each dose administered over $2-3 \mathrm{~h}$, imipenem-relebactam is given via a $30-\mathrm{min}$ infusion. Shorter infusion durations may be advocated in some institutional settings.

Zidebactam, a novel diazabicyclooctane $\beta$-lactamase inhibitor, serves two functions by preventing broad spectrum hydrolysis of cefepime and binding PBP2 [68, 69]. Currently it is in phase I of development. The combination theoretically works against $\mathrm{A}, \mathrm{C}$, and $\mathrm{D} \beta$-lactamase Enterobacterales with additional in vitro restored function to some group B $\beta$-lactamase Enterobacterales, drug-resistant $P$. aeruginosa, and drug-resistant $A$. baumannii [69]. In vivo cefepime-zidebactam has shown 1 to greater than $3 \log$ reductions of growth, and $1.6 \mathrm{log}$ reductions in growth for Enterobacterales and P. aeruginosa, respectively [68]. Further research is warranted for zidebactam as it may be the next breakthrough novel antimicrobial.

There are limited data comparing $\beta$-lactam $/ \beta$-lactamase inhibitors in the treatment of CRE which makes it difficult to determine a superior drug with overlapping susceptibilities. At least one BL/BLI which covers carbapenemase-producing Enterobacterales and P. aeruginosa should be on each hospital formulary. Local resistance patterns to Enterobacterales and Pseudomonas aeruginosa as well as the availability of microbiologic testing should be taken into account when making formulary decisions.

\section{Cefiderocol}

Coined as the "Trojan horse" antimicrobial, cefiderocol uses a siderophore to hijack bacteria's iron uptake system and enter the cell to disrupt cell wall synthesis [70-72]. Cefiderocol is stable and potent against all Ambler group $\beta$-lactamases in vitro, including MBLs, without the addition of a $\beta$ lactamase inhibitor $[70,73,74]$. In vivo and healthy volunteer studies have demonstrated adequate tissue concentration into epithelial lining fluid making it a promising therapy in bacterial pneumonia [73, 75]. Cefiderocol responded well against Gram-negative isolates of Klebsiella spp., P. aeruginosa, and A. baumannii with a greater than $3 \log$ bacterial growth reduction in a rat lung model. Similar results were seen in an immunocompromised murine thigh infection model with greater than $3 \log$ bacterial growth reduction against various Enterobacterales [74]. Cefiderocol is currently approved for complicated urinary tract infections as it was non-inferior to imipenem in a phase II trial. It is important to note that carbapenem-resistant isolates were excluded from this trial [76]. Cefiderocol is a welcomed novel antimicrobial for a 


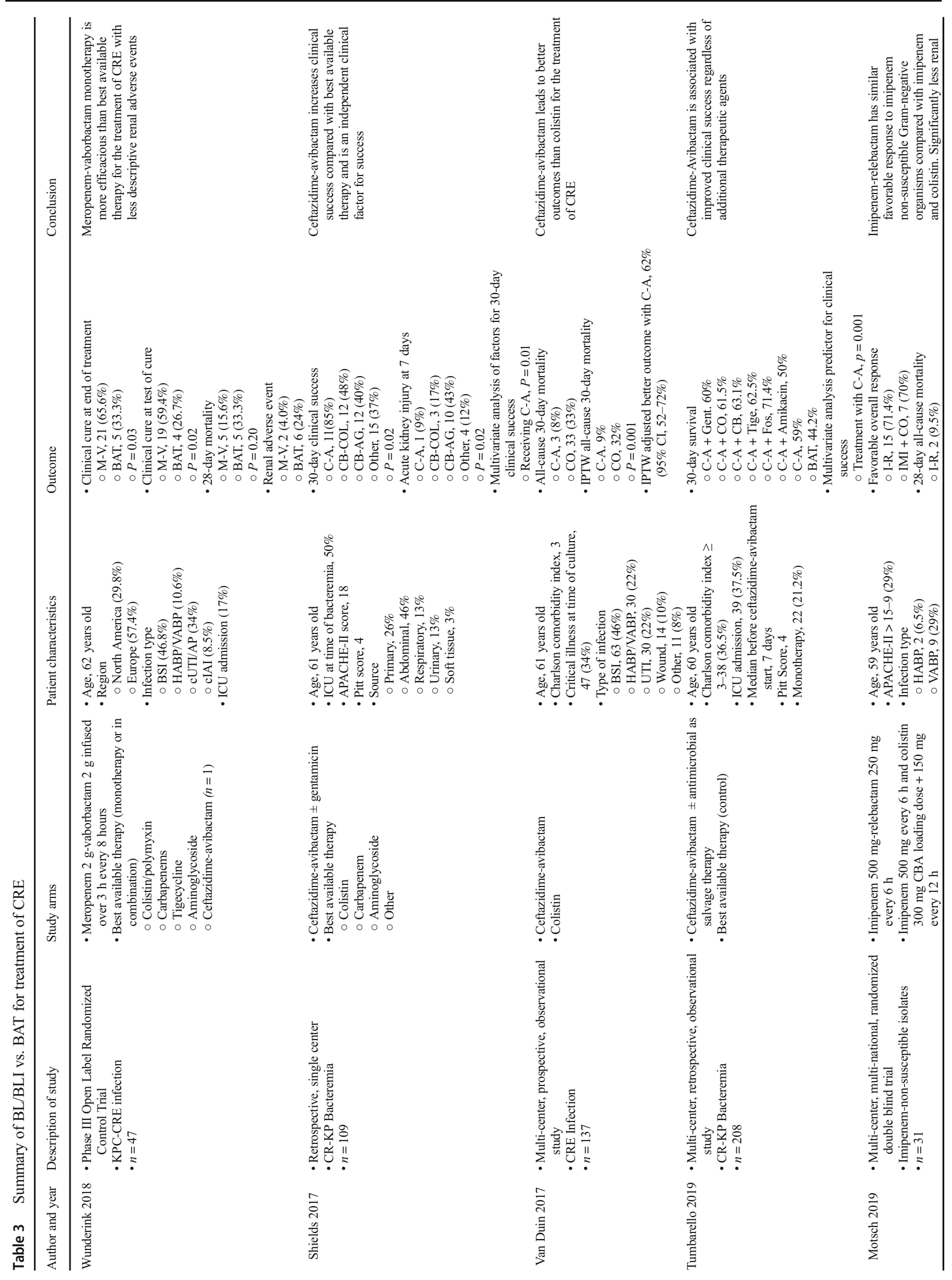


clinician's repertoire. Not only is this a novel mechanism of delivery, but also it may be one of the first monotherapy agents to effectively treat metallo- $\beta$-lactamase with minimal adverse events. Further clinical development will help determine the exact niche for cefiderocol.

\section{Fosfomycin}

IV fosfomycin is a treatment gaining interest in the USA for the treatment of various MDR organisms due to high rates of susceptibility and tolerability [77, 78]. A small prospective study including 11 critically ill patients evaluated the use of IV fosfomycin in combination against hospital-acquired carbapenem-resistant Enterobacterales and found that all-cause mortality rates $(18.2 \%)$ were lower than other best available therapies [79]. A potential fail point of this therapy is development of resistance during treatment when used as monotherapy [80]. The USA lags behind a majority of other countries as oral fosfomycin is the only formulation available at this moment; however, an IV formulation is currently under development [81]. Fosfomycin will likely be combined with additional agents for its additive and synergistic effects. Avery and colleagues evaluated combination fosfomycin against various CPE isolates using the E-test method [82]. Fosfomycin had no antagonism against any tested antimicrobial and had at least one isolate that was additive making it a potential option for treating multidrug-resistant organisms.

\section{Monotherapy vs. Combination and Colistin or BL/BLI as Backbone of Therapy}

The treatment of a non-metallo carbapenemase-producing Enterobacterales with monotherapy or combination has been a topic of interest for many clinicians now that novel $\beta$-lactam/ $\beta$-lactamase inhibitors have been introduced into clinical practice. Historically, the high mortality rates of polymyxins due to poor microbiologic potency and less than optimal drug exposures that have been limited by toxicity have mandated the use of combination therapy in severely ill patients. A large retrospective cohort study evaluated the treatment of bloodstream infections either with monotherapy or combination therapy [83]. Three hundred forty-three subjects were stratified into groups based on severity of illness with a primary endpoint of overall mortality. There were no statistical differences among combined cohorts (HR 0.76, 95\% CI 0.53-1.08) and the low mortality score cohort (HR $1.18,95 \%$ CI 0.62 2.23); however, subjects identified as a high risk of mortality had a statistically significant decrease in overall mortality (HR $0.6,95 \%$ CI 0.39-0.93). It is important to note the above study did not include novel therapies like BL/BLIs which should take over as the mainstay of CPO therapy. The shift away from 


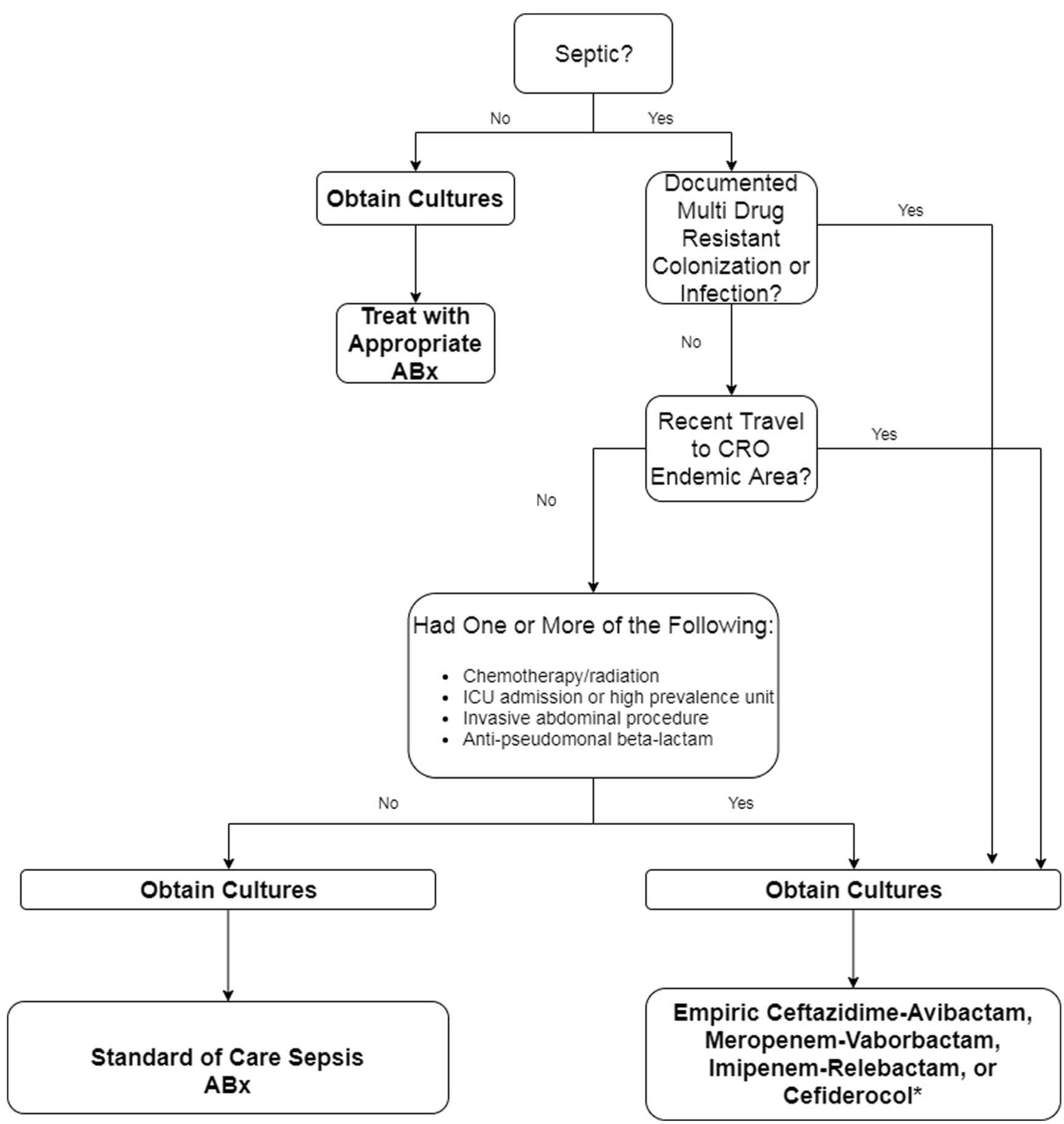

Fig. 1 Algorithm for the empiric therapy of the critically ill septic patient. Choose empiric coverage based on patient specific factors, local epidemiology, and concordant resistance. $\mathrm{ABX}$, antimicrobial, $\mathrm{CRO}$, carbapenem-resistant organism

using nephrotoxic agents with unpredictable pharmacokinetics has reopened the debate of which agent is superior. There is a growing amount of literature demonstrating ceftazidimeavibactam, imipenem-relebactam, and meropenemvaborbactam lead to better treatment outcomes with less adverse events compared with polymyxin best available therapy regimens [49, 52-54, 58, 67]. A summary comparing BL/BLI therapy to traditional colistin-based therapy can be seen in
Table 3. If an organism is susceptible to a novel $\beta$-lactam $/ \beta$ lactamase inhibitor, then that agent may be considered for monotherapy because these compounds have demonstrated efficacy in the setting of severe infections. Even with novel agents demonstrating superiority over colistin, prescribing habits are slow to change. A recent publication by Clancy and colleagues show that polymyxin and colistin are still the most prescribed antimicrobials for CRE infections which is 
deeply concerning [84]. Gaining access to novel agents to treat CRE should be a priority for all clinicians as these $\beta$ lactam/ $\beta$-lactamase inhibitors have been shown to improve outcomes and reduced toxicities relative to polymyxin-based regimens.

\section{Empiric Therapy}

Another carbapenemase treatment dogma often overshadowed in literature is when to use empiric therapy which covers carbapenemase. A prospective observational cohort study conducted by Gianella and colleagues evaluated risk factors for development of CRE bloodstream infection from previously colonized subjects and found ICU admission, invasive abdominal procedures, chemotherapy/radiation, and multiple CRE colonization sites independently increased risk [85]. Another study conducted in Israel demonstrated that clinically significant increased risks in carbapenem-resistant colonized patients developing infection were treated with antipseudomonal penicillins [86]. In an effort to maximize the probability of early appropriate therapy in patients at risk for resistant pathogens, clinicians need to become increasingly comfortable with the antimicrobial spectrum, pharmacokinetic and toxicodynamic profiles of the novel therapies discussed so that optimal empiric therapy can be initiated at the onset of infection (Fig. 1). Unfortunately a single source of antimicrobial susceptibility data for the agents included in this review is not available and thus comparative assessments based on percent susceptible collated from multiple sources are potentially misleading for CRE due to differing genotypic profiles across the globe. As such, these challenges highlight the importance of local susceptibility testing to determine the most suitable agent(s) for clinical practice in any given region. While the use of novel broad-spectrum antimicrobial agents may be warranted in the setting of initial empirical therapy for the septic patient, great effort should be taken to utilize the available diagnostic techniques (i.e., invasive sampling strategies, molecular tests) to identify the causative pathogen(s) and thus allow a rapid, narrow targeted approach to treatment.

\section{Conclusion}

The rapid emergence of carbapenemase-producing organisms is a major concern for human health. It is of utmost importance that clinicians are familiar with what antimicrobials can be used to treat serious infections like carbapenem-resistant Enterobacterales as well as the evolving enzymology associated with these pathogens. With the introduction of new $\beta$ lactam $/ \beta$-lactamase inhibitors and the improved clinical outcomes associated with these compounds, a shift away from the less efficacious, more toxic polymyxin-based regimens appears completely warranted. While the $\beta$-lactam $/ \beta$ lactamase inhibitors will play an important role moving forward, other parenteral agents such as plazomicin, fosfomycin, and cefiderocol show great promise and are welcomed options for treating multidrug-resistant organisms.

\section{Compliance with Ethical Standards}

Conflict of Interest MJL has no conflicts of interest to declare.

DPN is a consultant, speaker's bureau member, or have received research funding from Allergan, Bayer, Cepheid, Merck, Melinta, Pfizer, Wockhardt, Shionogi, and Tetraphase.

Human and Animal Rights and Informed Consent This article does not contain any studies with human or animal subjects performed by any of the authors.

\section{References}

1. World Health Organization. Top ten threats to global health in 2019. 2019 [cited 2019]; Available from: https://www.who.int/ emergencies/ten-threats-to-global-health-in-2019. Accessed Nov 2019.

2. Center for Disease Control and Prevention. Antibiotic resistance threats in the United States. 2013 [cited 2019]; Available from: https://www.cdc.gov/drugresistance/threat-report-2013/pdf/arthreats-2013-508.pdf. Accessed Nov 2019.

3. Center for Disease Control and Prevention. Tracking CRE HAI. 2019 May, 9, 2019; Available from: https://www.cdc.gov/hai/ organisms/cre/trackingcre.html. Accessed Nov 2019.

4. Thaden JT, Pogue JM, Kaye KS. Role of newer and re-emerging older agents in the treatment of infections caused by carbapenemresistant Enterobacteriaceae. Virulence. 2017;8(4):403-16.

5. Trecarichi EM, et al. Bloodstream infections caused by Klebsiella pneumoniae in onco-hematological patients: clinical impact of carbapenem resistance in a multicentre prospective survey. Am J Hematol. 2016;91(11):1076-81.

6. Facility guidance for control of carbapenem-resistant Enterobacteriaceae (CRE). 2015 November 2015; Available from: https://www.cdc.gov/hai/pdfs/cre/CRE-guidance-508.pdf. Accessed Nov 2019

7. Logan LK, Weinstein RA. The epidemiology of carbapenemresistant Enterobacteriaceae: the impact and evolution of a global menace. J Infect Dis. 2017;215(suppl_1):S28-s36.

8. Tängdén T, Adler M, Cars O, Sandegren L, Löwdin E. Frequent emergence of porin-deficient subpopulations with reduced carbapenem susceptibility in ESBL-producing Escherichia coli during exposure to ertapenem in an in vitro pharmacokinetic model. J Antimicrob Chemother. 2013;68(6):1319-26.

9. Yang F-C, et al. Characterization of Ertapenem-resistant Enterobacter cloacae in a Taiwanese university hospital. J Clin Microbiol. 2012;50(2):223-6.

10. Tamma PD, Goodman KE, Harris AD, Tekle T, Roberts A, Taiwo A, et al. Comparing the outcomes of patients with carbapenemaseproducing and non-carbapenemase-producing carbapenemresistant Enterobacteriaceae bacteremia. Clin Infect Dis. 2016;64(3):257-64.

11. Stewart A, et al. Treatment of infections by OXA-48-producing Enterobacteriaceae. Antimicrob Agents Chemother. 2018;62(11): e01195-18. 
12. Walther-Rasmussen J, Høiby N. OXA-type carbapenemases. J Antimicrob Chemother. 2006;57(3):373-83.

13. Potter RF, D'Souza AW, Dantas G. The rapid spread of carbapenem-resistant Enterobacteriaceae. Drug Resist Updat. 2016;29:30-46.

14. van Duin D, Doi Y. The global epidemiology of carbapenemaseproducing Enterobacteriaceae. Virulence. 2017;8(4):460-9.

15. Walsh TR. The emergence and implications of metallo- $\beta$ lactamases in Gram-negative bacteria. Clin Microbiol Infect. 2005;11:2-9.

16. Yigit H, Queenan AM, Anderson GJ, Domenech-Sanchez A, Biddle JW, Steward CD, et al. Novel carbapenem-hydrolyzing $\beta$ lactamase, KPC-1, from a carbapenem-resistant strain of Klebsiella pneumoniae. Antimicrob Agents Chemother. 2001;45(4):1151-61.

17. Yong $\mathrm{D}$, et al. Characterization of a new metallo- $\beta$-lactamase gene, Bla-NDM-1, and a novel erythromycin esterase gene carried on a unique genetic structure in Klebsiella pneumoniae sequence type 14 from India. Antimicrob Agents Chemother. 2009;53(12):5046-54.

18. Aloush V, Navon-Venezia S, Seigman-Igra Y, Cabili S, Carmeli Y. Multidrug-resistant Pseudomonas aeruginosa: risk factors and clinical impact. Antimicrob Agents Chemother. 2006;50(1):43-8.

19. Nation RL, Velkov T, Li J. Colistin and polymyxin B: peas in a pod, or chalk and cheese? Clin Infect Dis. 2014;59(1):88-94.

20. Tsuji BT, Pogue JM, Zavascki AP, Paul M, Daikos GL, Forrest A, et al. International Consensus guidelines for the optimal use of the polymyxins: endorsed by the American College of Clinical Pharmacy (ACCP), European Society of Clinical Microbiology and Infectious Diseases (ESCMID), Infectious Diseases Society of America (IDSA), International Society for Anti-infective Pharmacology (ISAP), Society of Critical Care Medicine (SCCM), and Society of Infectious Diseases Pharmacists (SIDP). Pharmacotherapy. 2019;39(1):10-39.

21. Capone A, Giannella M, Fortini D, Giordano A, Meledandri M, Ballardini M, et al. High rate of colistin resistance among patients with carbapenem-resistant Klebsiella pneumoniae infection accounts for an excess of mortality. Clin Microbiol Infect. 2013;19(1):E23-e30.

22. Markou N, Apostolakos H, Koumoudiou C, Athanasiou M, Koutsoukou A, Alamanos I, et al. Intravenous colistin in the treatment of sepsis from multiresistant Gram-negative bacilli in critically ill patients. Crit Care. 2003;7(5):R78-83.

23. Perez F, et al. Polymyxins: to combine or not to combine? Antibiotics (Basel). 2019;8(2).

24. Qureshi ZA, Paterson DL, Potoski BA, Kilayko MC, Sandovsky G, Sordillo E, et al. Treatment outcome of bacteremia due to KPCproducing Klebsiella pneumoniae: superiority of combination antimicrobial regimens. Antimicrob Agents Chemother. 2012;56(4): 2108-13.

25. Sandri AM, Landersdorfer CB, Jacob J, Boniatti MM, Dalarosa MG, Falci DR, et al. Population pharmacokinetics of intravenous polymyxin B in critically ill patients: implications for selection of dosage regimens. Clin Infect Dis. 2013;57(4):524-31.

26. Thamlikitkul V, et al. Dosing and pharmacokinetics of polymyxin B in patients with renal insufficiency. Antimicrob Agents Chemother. 2017;61(1):e01337-16.

27. Krause KM, et al. Aminoglycosides: an overview. Cold Spring Harb Perspect Med. 2016;6(6).

28. Nicolau DP, et al. Experience with a once-daily aminoglycoside program administered to 2,184 adult patients. Antimicrob Agents Chemother. 1995;39(3):650-5.

29. Doi Y, Arakawa Y. 16S ribosomal RNA methylation: emerging resistance mechanism against aminoglycosides. Clin Infect Dis. 2007;45(1):88-94.

30. Zhou Y, Yu H, Guo Q, Xu X, Ye X, Wu S, et al. Distribution of $16 \mathrm{~S}$ rRNA methylases among different species of Gram-negative bacilli with high-level resistance to aminoglycosides. Eur J Clin Microbiol Infect Dis. 2010;29(11):1349-53.

31. Abdelraouf $\mathrm{K}$, et al. In vivo efficacy of plazomicin alone or in combination with meropenem or tigecycline against Enterobacteriaceae isolates exhibiting various resistance mechanisms in an immunocompetent murine septicemia model. Antimicrob Agents Chemother. 2018;62(8):e01074-18.

32. Castanheira $M$, et al. In vitro activity of plazomicin against Gramnegative and Gram-positive isolates collected from U.S. hospitals and comparative activities of aminoglycosides against carbapenemresistant Enterobacteriaceae and isolates carrying carbapenemase genes. Antimicrob Agents Chemother. 2018;62(8).

33. Zhang Y, Kashikar A, Bush K. In vitro activity of plazomicin against beta-lactamase-producing carbapenem-resistant Enterobacteriaceae (CRE). J Antimicrob Chemother. 2017;72(10): 2792-5.

34. McKinnell JA, et al. Plazomicin for infections caused by carbapenem-resistant Enterobacteriaceae. N Engl J Med. 2019;380(8):791-3.

35. Kuti JL, et al. Evaluation of plazomicin, tigecycline, and meropenem pharmacodynamic exposure against carbapenemresistant Enterobacteriaceae in patients with bloodstream infection or hospital-acquired/ventilator-associated pneumonia from the CARE study (ACHN-490-007). Infect Dis Ther. 2019.

36. Asempa TE, et al. A simulated application of the Hartford Hospital aminoglycoside dosing nomogram for plazomicin dosing interval selection in patients with serious infections caused by carbapenemresistant Enterobacterales. Clin Ther. 2019.

37. Asempa TE, et al. Application of the Hartford Hospital nomogram for plazomicin dosing interval selection in patients with complicated urinary tract infection. Antimicrob Agents Chemother. 2019;63(10):e00148-19.

38. Bulik CC, Nicolau DP. Double-carbapenem therapy for carbapenemase-producing Klebsiella pneumoniae. Antimicrob Agents Chemother. 2011;55(6):3002-4.

39. Cprek JB, Gallagher JC. Ertapenem-containing double-carbapenem therapy for treatment of infections caused by carbapenem-resistant Klebsiella pneumoniae. Antimicrob Agents Chemother. 2016;60(1):669-73.

40. De Pascale G, et al. Double carbapenem as a rescue strategy for the treatment of severe carbapenemase-producing Klebsiella pneumoniae infections: a two-center, matched case-control study. Crit Care. 2017;21(1):173.

41. Giamarellou H, Galani L, Baziaka F, Karaiskos I. Effectiveness of a double-carbapenem regimen for infections in humans due to carbapenemase-producing pandrug-resistant Klebsiella pneumoniae. Antimicrob Agents Chemother. 2013;57(5):2388-90.

42. Souli M, Karaiskos I, Masgala A, Galani L, Barmpouti E, Giamarellou H. Double-carbapenem combination as salvage therapy for untreatable infections by KPC-2-producing Klebsiella pneumoniae. Eur J Clin Microbiol Infect Dis. 2017;36(7):1305-15.

43. Coleman K. Diazabicyclooctanes (DBOs): a potent new class of non-beta-lactam beta-lactamase inhibitors. Curr Opin Microbiol. 2011;14(5):550-5.

44. Kazmierczak KM, et al. In vitro activity of ceftazidime-avibactam and aztreonam-avibactam against OXA-48-carrying Enterobacteriaceae isolated as part of the international network for optimal resistance monitoring (INFORM) global surveillance program from 2012 to 2015. Antimicrob Agents Chemother. 2018;62(12):e00592-18.

45. Karlowsky JA, et al. In vitro activity of aztreonam-avibactam against Enterobacteriaceae and Pseudomonas aeruginosa isolated by clinical laboratories in 40 countries from 2012 to 2015 . Antimicrob Agents Chemother. 2017;61(9):e00472-17.

46. Marshall S, et al. Can ceftazidime-avibactam and aztreonam overcome $\beta$-lactam resistance conferred by metallo- $\beta$-lactamases in 
Enterobacteriaceae? Antimicrob Agents Chemother. 2017;61(4): e02243-16.

47. Wenzler E, Deraedt MF, Harrington AT, Danizger LH. Synergistic activity of ceftazidime-avibactam and aztreonam against serine and metallo-beta-lactamase-producing Gram-negative pathogens. Diagn Microbiol Infect Dis. 2017;88(4):352-4.

48. Castón JJ, Lacort-Peralta I, Martín-Dávila P, Loeches B, Tabares S, Temkin L, et al. Clinical efficacy of ceftazidime/avibactam versus other active agents for the treatment of bacteremia due to carbapenemase-producing Enterobacteriaceae in hematologic patients. Int J Infect Dis. 2017;59:118-23.

49. Shields RK, et al. Ceftazidime-avibactam is superior to other treatment regimens against carbapenem-resistant Klebsiella pneumoniae bacteremia. Antimicrob Agents Chemother. 2017;61(8):e00883-17.

50. Sousa A, Pérez-Rodríguez MT, Soto A, Rodríguez L, PérezLandeiro A, Martínez-Lamas L, et al. Effectiveness of ceftazidime/avibactam as salvage therapy for treatment of infections due to OXA-48 carbapenemase-producing Enterobacteriaceae. J Antimicrob Chemother. 2018;73(11):31705.

51. Temkin E, et al. Ceftazidime-avibactam as salvage therapy for infections caused by carbapenem-resistant organisms. Antimicrob Agents Chemother. 2017;61(2):e01964-16.

52. Tumbarello $\mathrm{M}$, et al. Efficacy of ceftazidime-avibactam salvage therapy in patients with infections caused by Klebsiella pneumoniae Carbapenemase-producing K. pneumoniae. Clin Infect Dis. 2018;68(3):355-64.

53. van Duin $\mathrm{D}$, et al. Colistin versus ceftazidime-avibactam in the treatment of infections due to carbapenem-resistant Enterobacteriaceae. Clin Infect Dis. 2017;66(2):163-71.

54. Shields RK, Potoski BA, Haidar G, Hao B, Doi Y, Chen L, et al. Clinical outcomes, drug toxicity, and emergence of ceftazidimeavibactam resistance among patients treated for carbapenemresistant Enterobacteriaceae infections. Clin Infect Dis. 2016;63(12):1615-8.

55. Lomovskaya $\mathrm{O}$, et al. Vaborbactam: spectrum of beta-lactamase inhibition and impact of resistance mechanisms on activity in Enterobacteriaceae. Antimicrob Agents Chemother. 2017;61(11): e01443-17.

56. Shields RK, et al. Pneumonia and renal replacement therapy are risk factors for ceftazidime-avibactam treatment failures and resistance among patients with carbapenem-resistant Enterobacteriaceae infections. Antimicrob Agents Chemother. 2018;62(5):e02497-17.

57. Sabet $\mathrm{M}$, et al. Activity of meropenem-vaborbactam in mouse models of infection due to KPC-producing carbapenem-resistant Enterobacteriaceae. Antimicrob Agents Chemother. 2018;62(1): e01446-17.

58. Wunderink RG, et al. Effect and safety of meropenem-vaborbactam versus best-available therapy in patients with carbapenem-resistant Enterobacteriaceae infections: the TANGO II randomized clinical trial. Infect Dis Ther. 2018;7(4):439-55.

59. CLSI. Performance standards for antimicrobial susceptibility testing. 29th ed. Clinical and Laboratory Standards Institute: Wayne; 2019.

60. Canver MC, et al. Activity of imipenem-relebactam and comparator agents against genetically characterized isolates of carbapenemresistant Enterobacteriaceae. Antimicrob Agents Chemother. 2019.

61. Zhanel GG, et al. Imipenem-relebactam and meropenemvaborbactam: two novel carbapenem-beta-lactamase inhibitor combinations. Drugs. 2018;78(1):65-98.

62. Asempa TE, Nicolau DP, Kuti JL. Activity of imipenem-relebactam alone or in combination with amikacin or colistin against Pseudomonas aeruginosa. Antimicrob Agents Chemother. 2019;63(9):e00997-19.
63. Haidar $\mathrm{G}$, et al. Identifying spectra of activity and therapeutic niches for ceftazidime-avibactam and imipenem-relebactam against carbapenem-resistant Enterobacteriaceae. Antimicrob Agents Chemother. 2017;61(9):e00642-17.

64. Lob SH, et al. In vitro activity of imipenem-relebactam against Gram-negative ESKAPE pathogens isolated by clinical laboratories in the United States in 2015 (results from the SMART global surveillance program). Antimicrob Agents Chemother. 2017;61(6): e02209-16.

65. Lucasti C, Vasile L, Sandesc D, Venskutonis D, McLeroth P, Lala $\mathrm{M}$, et al. Phase 2, dose-ranging study of relebactam with imipenemcilastatin in subjects with complicated intra-abdominal infection. Antimicrob Agents Chemother. 2016;60(10):6234- 43.

66. Powles MA, et al. In vivo efficacy of relebactam (MK-7655) in combination with imipenem-cilastatin in murine infection models. Antimicrob Agents Chemother. 2018;62(8):e02577-17.

67. Motsch J, et al. RESTORE-IMI 1: a multicenter, randomized, double-blind trial comparing efficacy and safety of imipenem/ relebactam vs colistin plus imipenem in patients with imipenemnonsusceptible bacterial infections. Clin Infect Dis. 2019.

68. Monogue ML, et al. In vivo efficacy of WCK 5222 (cefepimezidebactam) against multidrug-resistant Pseudomonas aeruginosa in the neutropenic murine thigh infection model. Antimicrob Agents Chemother. 2019;63(7):e00233-19.

69. Moya $B$, et al. In vitro and in vivo activities of $\beta$-lactams in combination with the novel $\beta$-lactam enhancers zidebactam and WCK 5153 against multidrug-resistant metallo- $\beta$-lactamase-producing Klebsiella pneumoniae. Antimicrob Agents Chemother. 2019;63(5):e00128-19.

70. Hackel MA, et al. In vitro activity of the siderophore cephalosporin, cefiderocol, against a recent collection of clinically relevant Gramnegative bacilli from North America and Europe, including carbapenem-nonsusceptible isolates (SIDERO-WT-2014 study). Antimicrob Agents Chemother. 2017;61(9):e00093-17.

71. Ito A, Nishikawa T, Matsumoto S, Yoshizawa H, Sato T, Nakamura $\mathrm{R}$, et al. Siderophore cephalosporin cefiderocol utilizes ferric iron transporter systems for antibacterial activity against Pseudomonas aeruginosa. Antimicrob Agents Chemother. 2016;60(12):7396401.

72. Katsube T, Echols R, Arjona Ferreira JC, Krenz HK, Berg JK, Galloway C. Cefiderocol, a siderophore cephalosporin for Gramnegative bacterial infections: pharmacokinetics and safety in subjects with renal impairment. J Clin Pharmacol. 2017;57(5):584-91.

73. Matsumoto S, et al. Efficacy of cefiderocol against carbapenemresistant Gram-negative bacilli in immunocompetent-rat respiratory tract infection models recreating human plasma pharmacokinetics. Antimicrob Agents Chemother. 2017;61(9):e00700-17.

74. Stainton SM, et al. Efficacy of humanized cefiderocol exposures over 72 hours against a diverse group of Gram-negative isolates in the neutropenic murine thigh infection model. Antimicrob Agents Chemother. 2019;63(2):e01040-18.

75. Katsube T, Saisho Y, Shimada J, Furuie H. Intrapulmonary pharmacokinetics of cefiderocol, a novel siderophore cephalosporin, in healthy adult subjects. J Antimicrob Chemother. 2019;74(7):19714.

76. Portsmouth S, van Veenhuyzen D, Echols R, Machida M, Ferreira JCA, Ariyasu M, et al. Cefiderocol versus imipenem-cilastatin for the treatment of complicated urinary tract infections caused by Gram-negative uropathogens: a phase 2, randomised, double-blind, non-inferiority trial. Lancet Infect Dis. 2018;18(12):1319-28.

77. Falagas ME, Vouloumanou EK, Samonis G, Vardakas KZ. Fosfomycin. Clin Microbiol Rev. 2016;29(2):321-47.

78. Pogue JM, et al. Fosfomycin activity versus carbapenem-resistant Enterobacteriaceae and vancomycin-resistant Enterococcus, Detroit, 2008-10. J Antibiot. 2013;66:625. 
79. Michalopoulos A, Virtzili S, Rafailidis P, Chalevelakis G, Damala $\mathrm{M}$, Falagas ME. Intravenous fosfomycin for the treatment of nosocomial infections caused by carbapenem-resistant Klebsiella pneumoniae in critically ill patients: a prospective evaluation. Clin Microbiol Infect. 2010;16(2):184-6.

80. Williams PCM, Waichungo J, Gordon NC, Sharland M, Murunga S, Kamau A, et al. The potential of fosfomycin for multi-drug resistant sepsis: an analysis of in vitro activity against invasive paediatric Gram-negative bacteria. J Med Microbiol. 2019;68(5): 711-9.

81. Kaye KS, et al. Fosfomycin for injection (ZTI-01) vs piperacillintazobactam (PIP-TAZ) for the treatment of complicated urinary tract infection (cUTI) including acute pyelonephritis (AP): ZEUS, a phase 2/3 randomized trial. Clin Infect Dis. 2019.

82. Avery LM, Sutherland CA, Nicolau DP. In vitro investigation of synergy among fosfomycin and parenteral antimicrobials against carbapenemase-producing Enterobacteriaceae. Diagn Microbiol Infect Dis. 2019

83. Gutiérrez-Gutiérrez B, Salamanca E, de Cueto M, Hsueh PR, Viale P, Paño-Pardo JR, et al. Effect of appropriate combination therapy on mortality of patients with bloodstream infections due to carbapenemase-producing Enterobacteriaceae (INCREMENT): a retrospective cohort study. Lancet Infect Dis. 2017;17(7):726-34.

84. Clancy CJ, et al. Estimating the treatment of carbapenem-resistant Enterobacteriaceae infections in the United States using antibiotic prescription data. Open Forum Infect Dis. 2019;6(8).

85. Giannella M, Trecarichi EM, de Rosa FG, del Bono V, Bassetti M, Lewis RE, et al. Risk factors for carbapenem-resistant Klebsiella pneumoniae bloodstream infection among rectal carriers: a prospective observational multicentre study. Clin Microbiol Infect. 2014;20(12):1357-62.

86. Borer A, Saidel-Odes L, Eskira S, Nativ R, Riesenberg K, LivshizRiven I, et al. Risk factors for developing clinical infection with carbapenem-resistant Klebsiella pneumoniae in hospital patients initially only colonized with carbapenem-resistant $\mathrm{K}$ pneumoniae. Am J Infect Control. 2012;40(5):421-5.

Publisher's Note Springer Nature remains neutral with regard to jurisdictional claims in published maps and institutional affiliations. 